\title{
ATLAS Silicon Pixel and Strip Detector: Commissioning with Cosmic Data
}

Maria Fiascaris (University of Oxford) on behalf of the ATLAS Collaboration

$11^{\text {th }}$ European Symposium on Semiconductor Detectors June $7^{\text {th }} 2009$ 


\section{Outline}

- ATLAS at the LHC

- The Inner Detector

- Pixel and SCT modules and read-out

- Commissioning:

- Connectivity and Services

- First Beam in ATLAS

- Cosmic Run

- Conclusions 


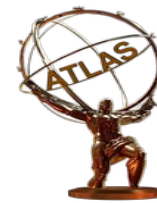 \\ The ATLAS Detector at the LHC}

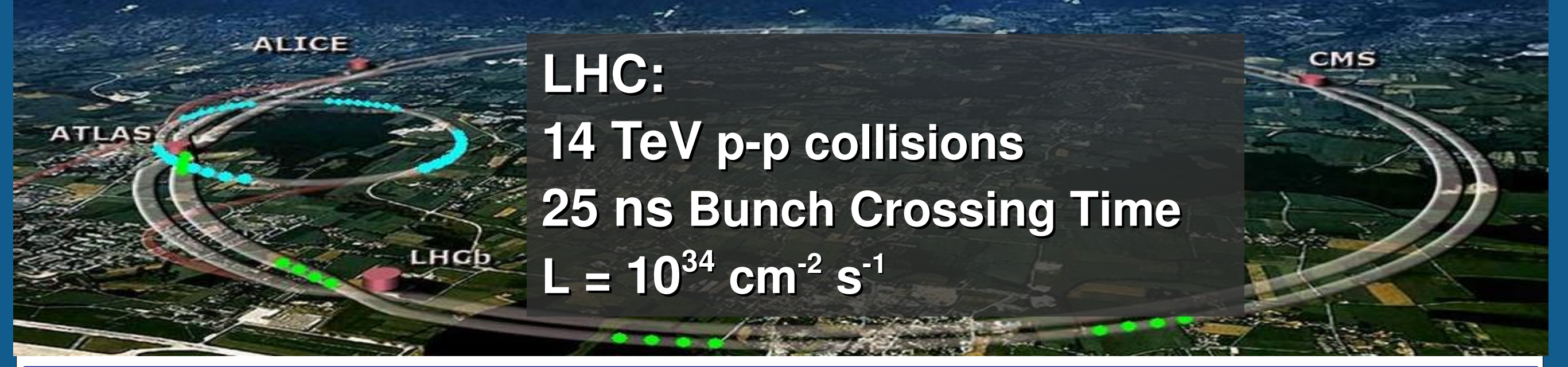

\section{ATLAS (General Purpose Detector)}

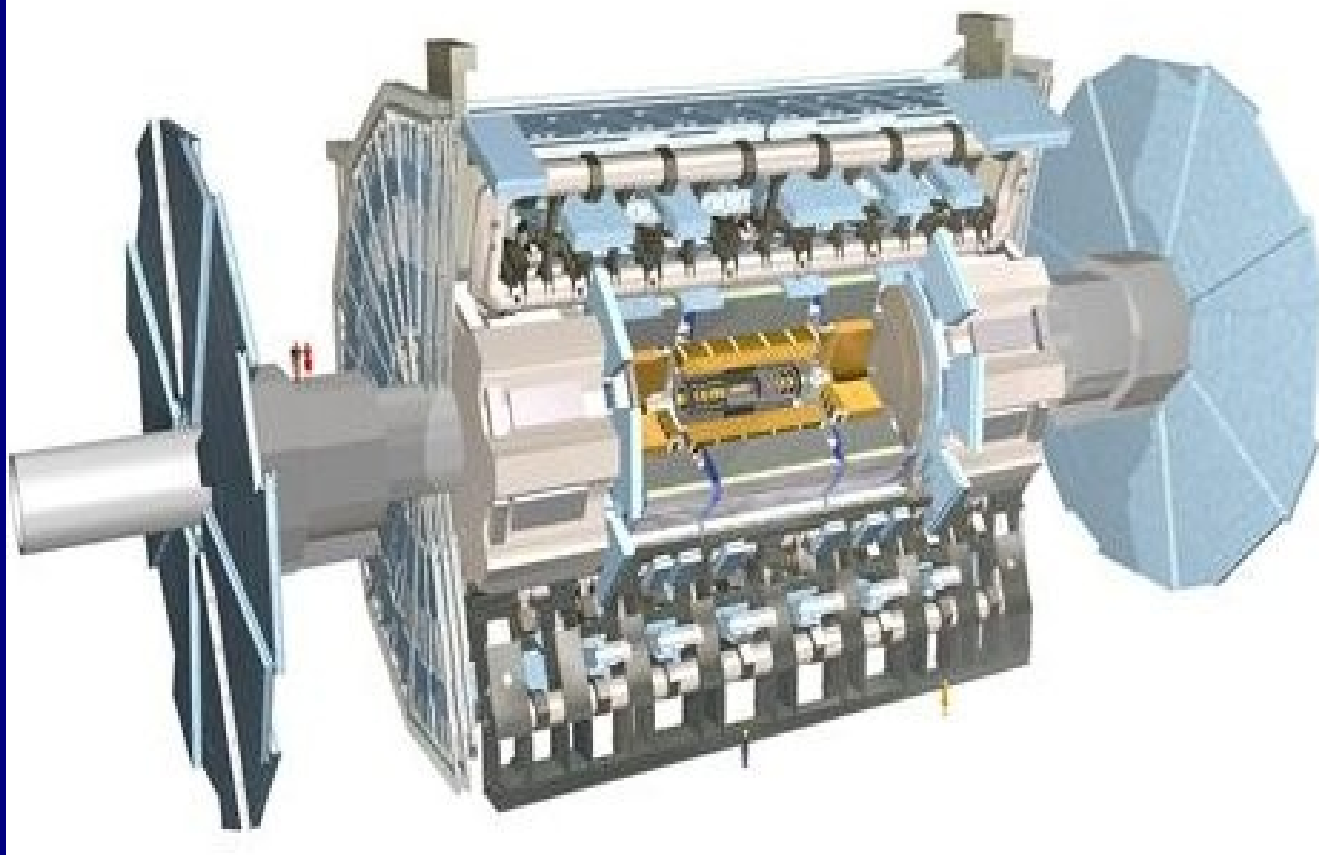

> Fast and radiation hard electronics and sensor elements

$>$ High Granularity

Inner Detector

$\left(|\eta|<2.5, \mathrm{p}_{\mathrm{T}}>0.5 \mathrm{GeV}\right)$ :

> Hermetic and robust pattern recognition

$>$ Excellent momentum resolution

$>$ Primary / secondary vertex measurement 


\section{The ATLAS Inner Detector}

In 2T solenoidal field:

$>$ Pixels

> Silicon Strip Detector (SCT)

$>$ Transition Radiation Tracker

> Radiation damage:

Si sensors cooled to -5 to $-10{ }^{\circ} \mathrm{C}$ $\left(\mathrm{C}_{3} \mathrm{~F}_{8}\right)$

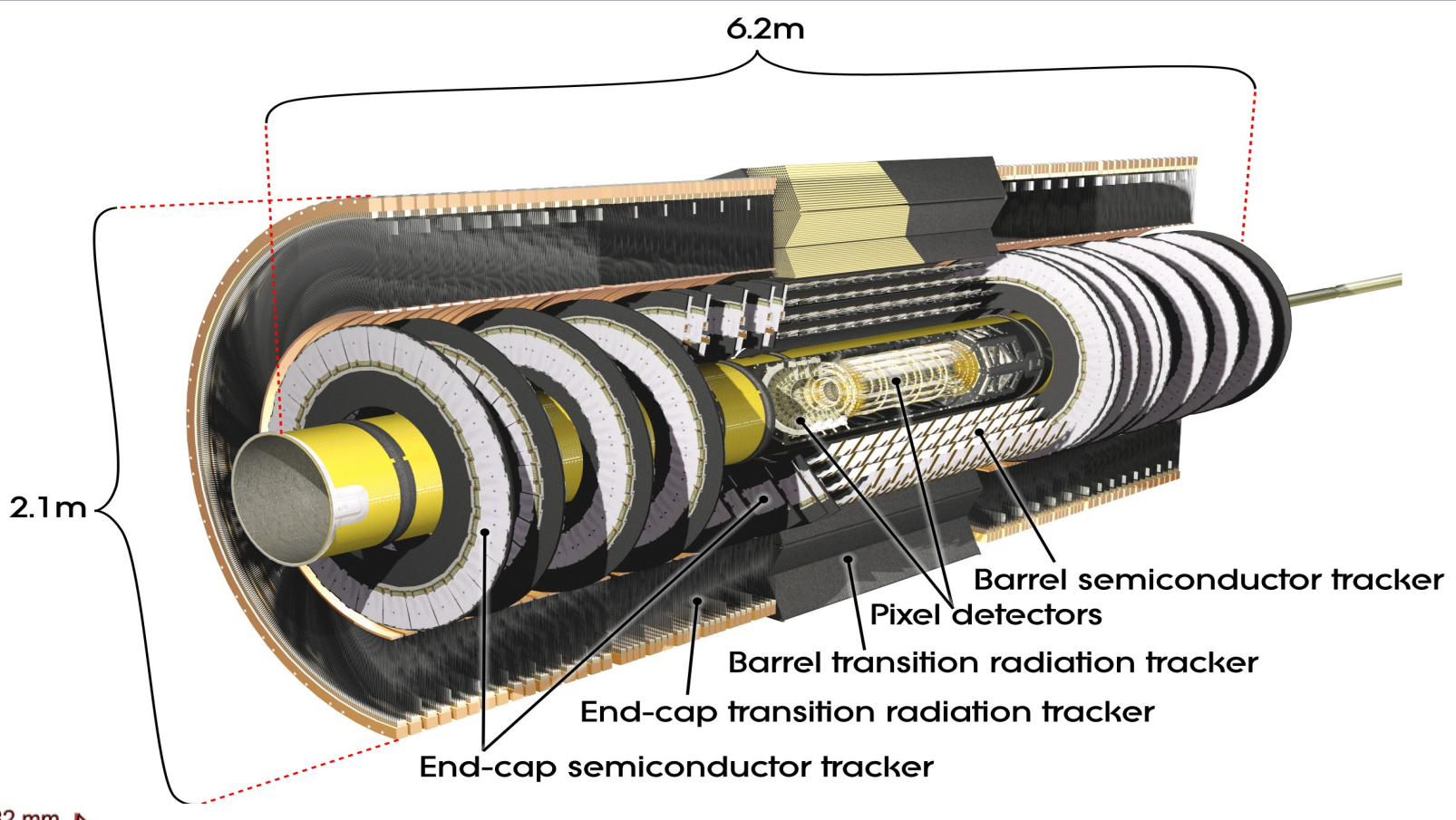

\section{Pixel Detector}

- Barrel Region : 3 layers

$>$ End-caps: 3 disks per side $>3$ space points

$>80.4 \mathrm{M}$ read-out channels

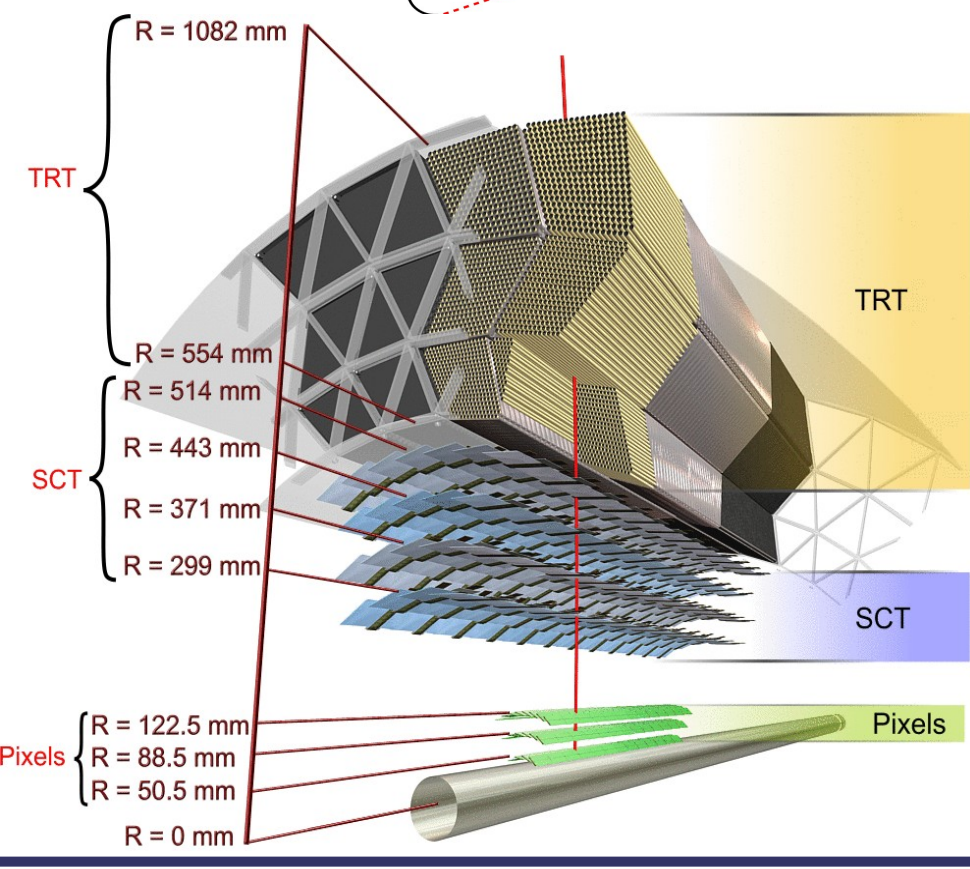

SCT

> Barrel Region : 4 layers

$>$ End-caps: 9 disks per side

$>4$ space points

$>6.3 \mathrm{M}$ read-out channels

$>63 \mathrm{~m}^{2}$ Silicon Area 


\section{Pixel Modules}

\section{Pixel Module:}

> Active surface: $6.08 \times 1.64 \mathrm{~cm}^{2}$

$>16$ FE chips bump bonded to sensor

> Flex hybrid with Module Control Chip

\section{Pixel Sensors:}

$>$ n-in-n technology (optimal radiation tolerance)

> Bulk depth $250 \mu \mathrm{m}$

> Typical pixel size: $50 \mu \mathrm{m}$ x $400 \mu \mathrm{m}$

> Accuracy: $10 \mu \mathrm{m}(\mathrm{R} \varphi), 115 \mu \mathrm{m}(\mathrm{z}, \mathrm{R})$

$>$ Radiation tolerance: $\mathrm{F}_{\text {neq }} \sim 1.1 \times 10^{15} \mathrm{~cm}^{-2}$ (10 years of operations)

$>$ Inner vertexing layer to be replaced after 3 years of operations

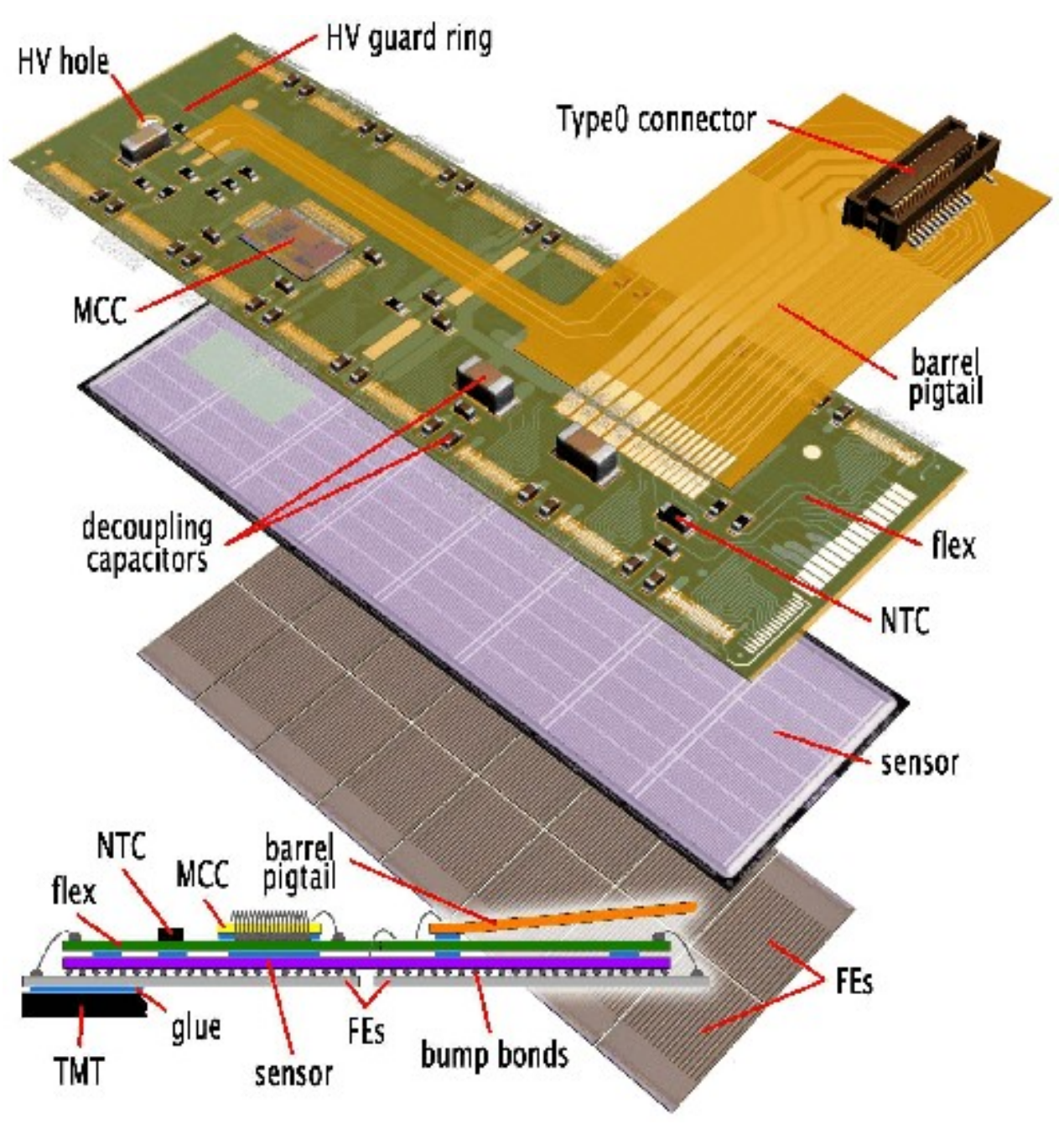




\section{SCT Modules}

\section{SCT Module:}

$>2$ planes of $\mathrm{Si}$ (2 sensors each)

Stereo angle of 40 mrad

> Barrel: identical modules

> End-caps: 3 different designs

\section{SCT Sensor:}

Single sided $p$-in-n technology

$>$ AC-coupled readout strips

> Bulk depth $285 \mu \mathrm{m}$

> Typical strip pitch $80 \mu \mathrm{m}$

$>$ Accuracy (from 2 wafers):

$17 \mu \mathrm{m}(\mathrm{R} \varphi), 580 \mu \mathrm{m}(\mathrm{z}, \mathrm{R})$

> Radiation tolerance:

$\mathrm{F}_{\text {neq }} \sim 2 \times 10^{14} \mathrm{~cm}^{-2}$ (10 years of operations)

\section{Barrel Module}

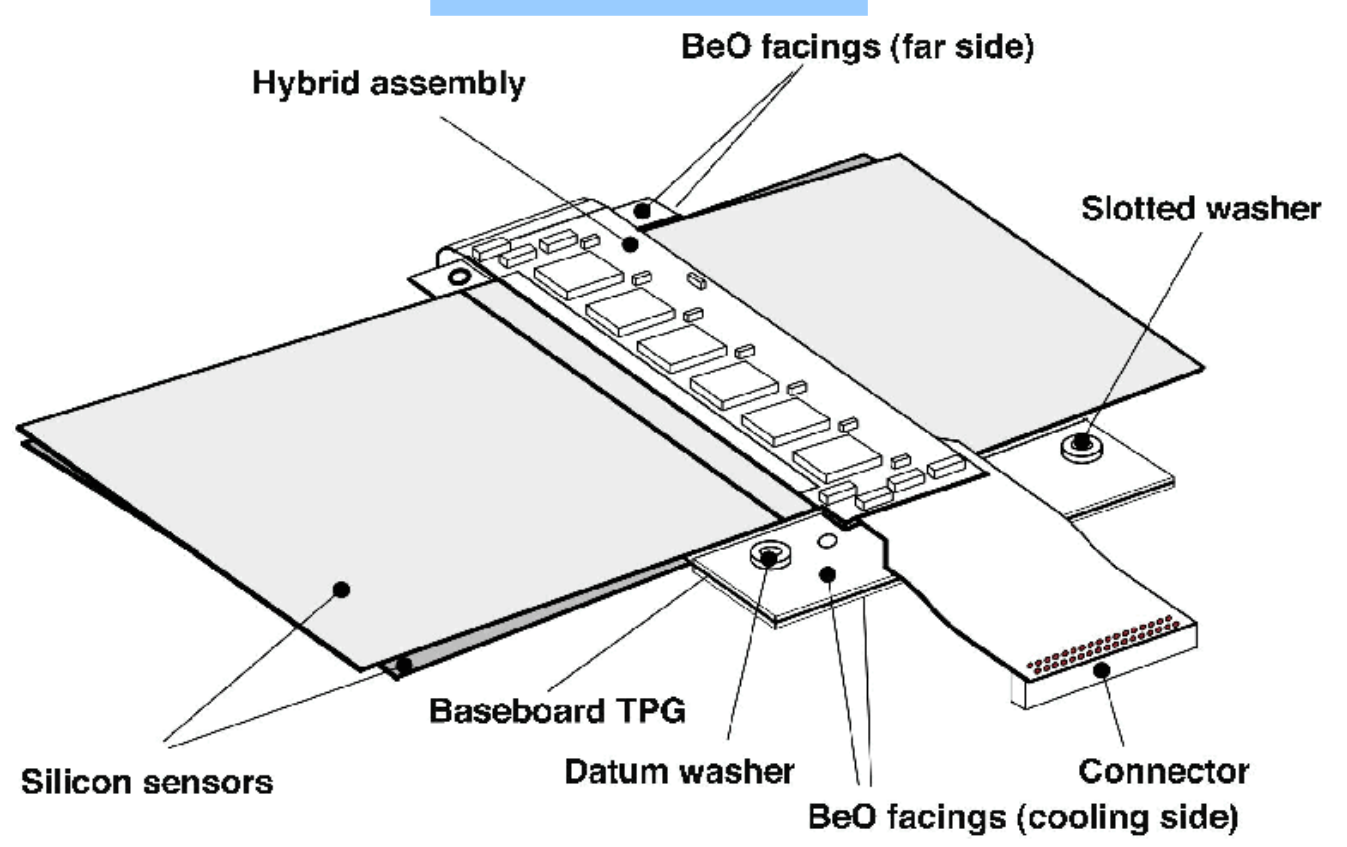

End-cap Modules
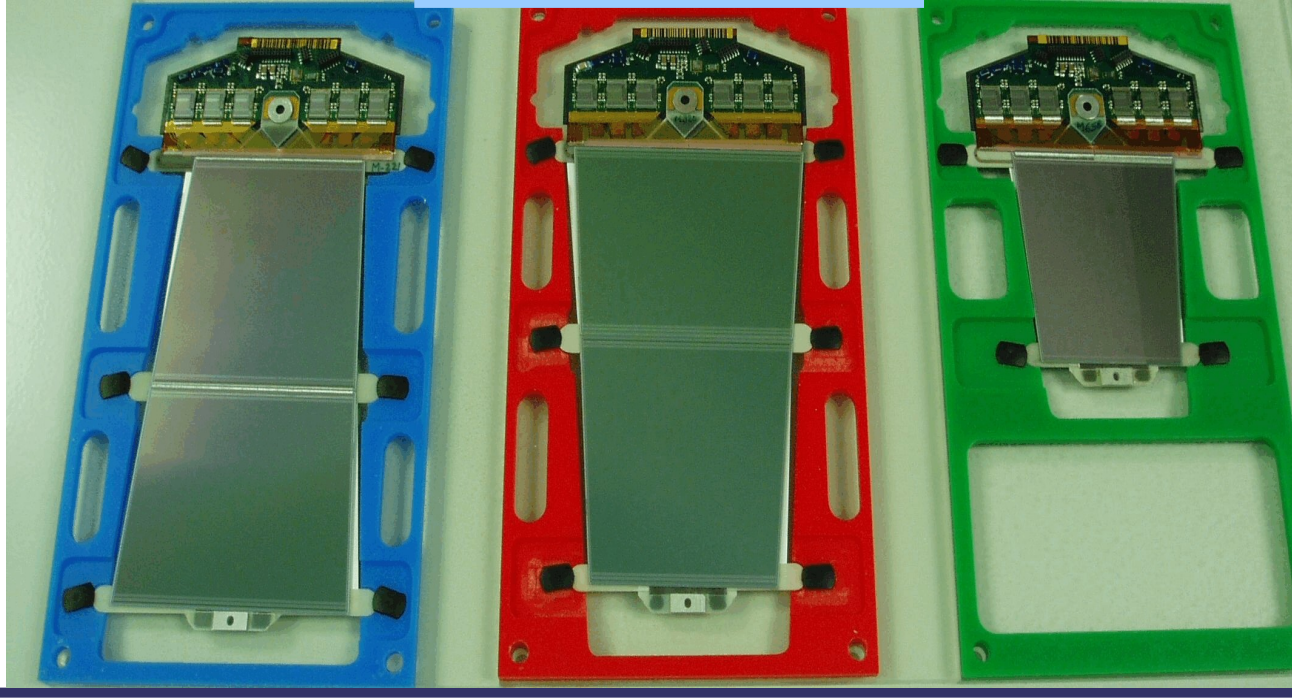


\section{Read-Out System}

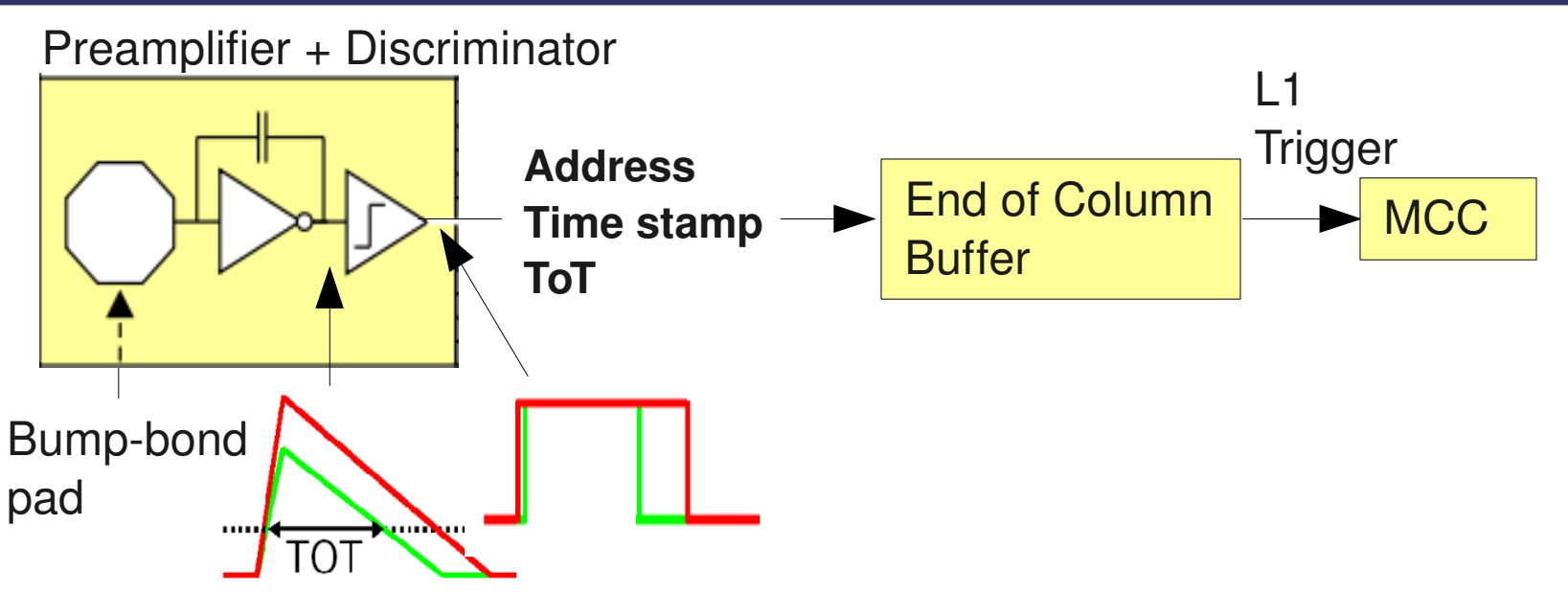

Pixels

$>16$ FE chips per module

$>0.25 \mu \mathrm{m}$ CMOS technology

> Digital read-out of 2880 pixel cells

> End-of-column readout buffers

\section{SCT}

$>12$ ABCD3T chips per module

$>$ DMILL technology

$>$ Binary read-out of 128 channels

$>$ 132-length binary pipeline

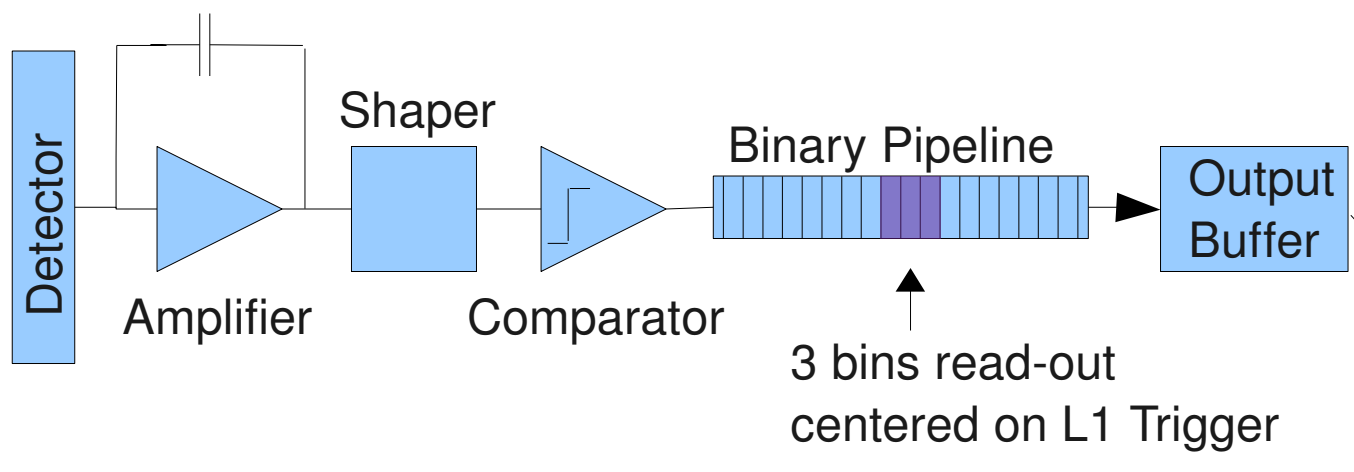

4

Data

Compression \& Readout

\section{Optical links}

> Data from on-detector to off-detector electronics

$>$ Trigger, timing, configuration data to the modules 


\section{Installation \& Commissioning}

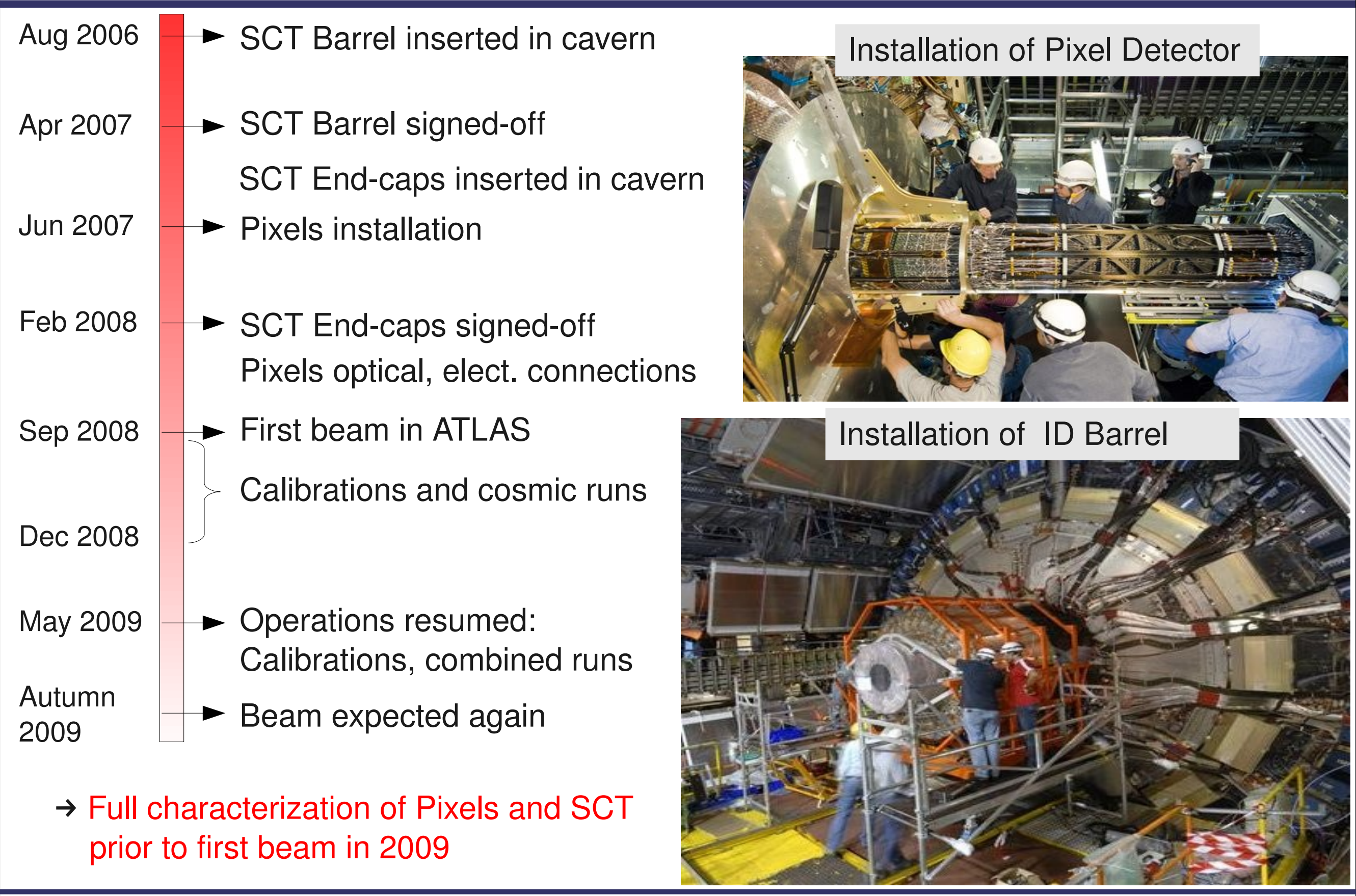




\section{Services and Connectivity}

\section{Evaporative Cooling System:}

$>$ Major problems encountered in 2007-2008 $\rightarrow$ fully operational from August 2008

> Upgrade in first months of 2009

$>$ Non-operational loops in autumn 2008:

$>3$ pixel loops (recoverable)

> 2 SCT End-cap loops due to a damaged cable (recovered, 23 modules)

a coolant leak (non recoverable, 13 modules)

\section{Optical Links}

> Test of optical connections and tuning of parameters

> Elevated mortality rate of TX plug-ins on off-detector boards observed

$>$ Evidence of electrostatic discharge during manufacture

$>$ TX plug-ins replacement with new production ongoing $\rightarrow$ to be completed by August 2009

\section{Power Supplies and Measurement of Environmental Variables}

> Electrical connections and power supplies

> Detector Control System: monitoring power supplies and environmental variables

> Fully commissioned in 2008 


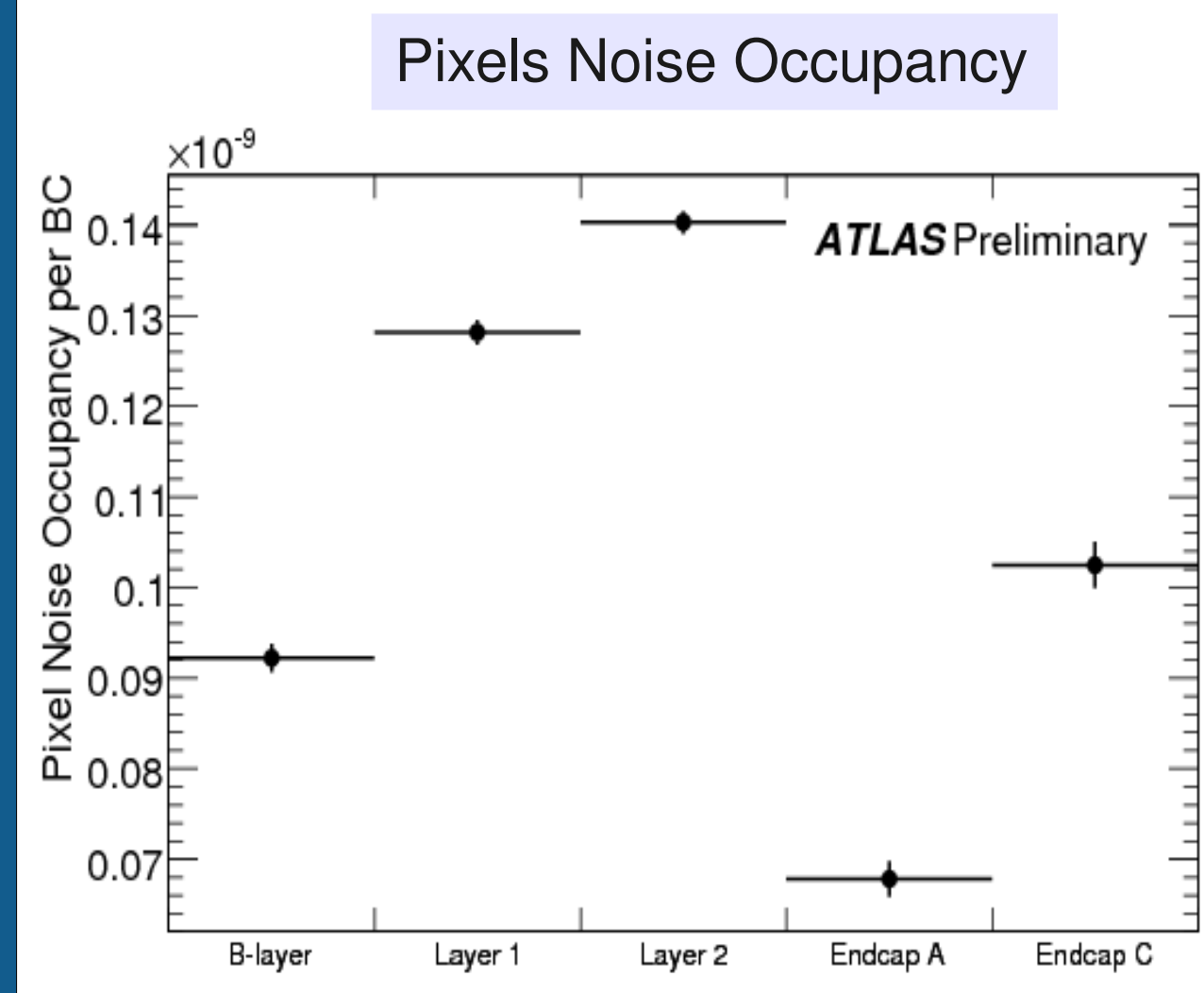

> After masking of noisy pixels $\left(10^{-4}\right)$

$>$ Noise occupancy $\sim 10^{-10}$

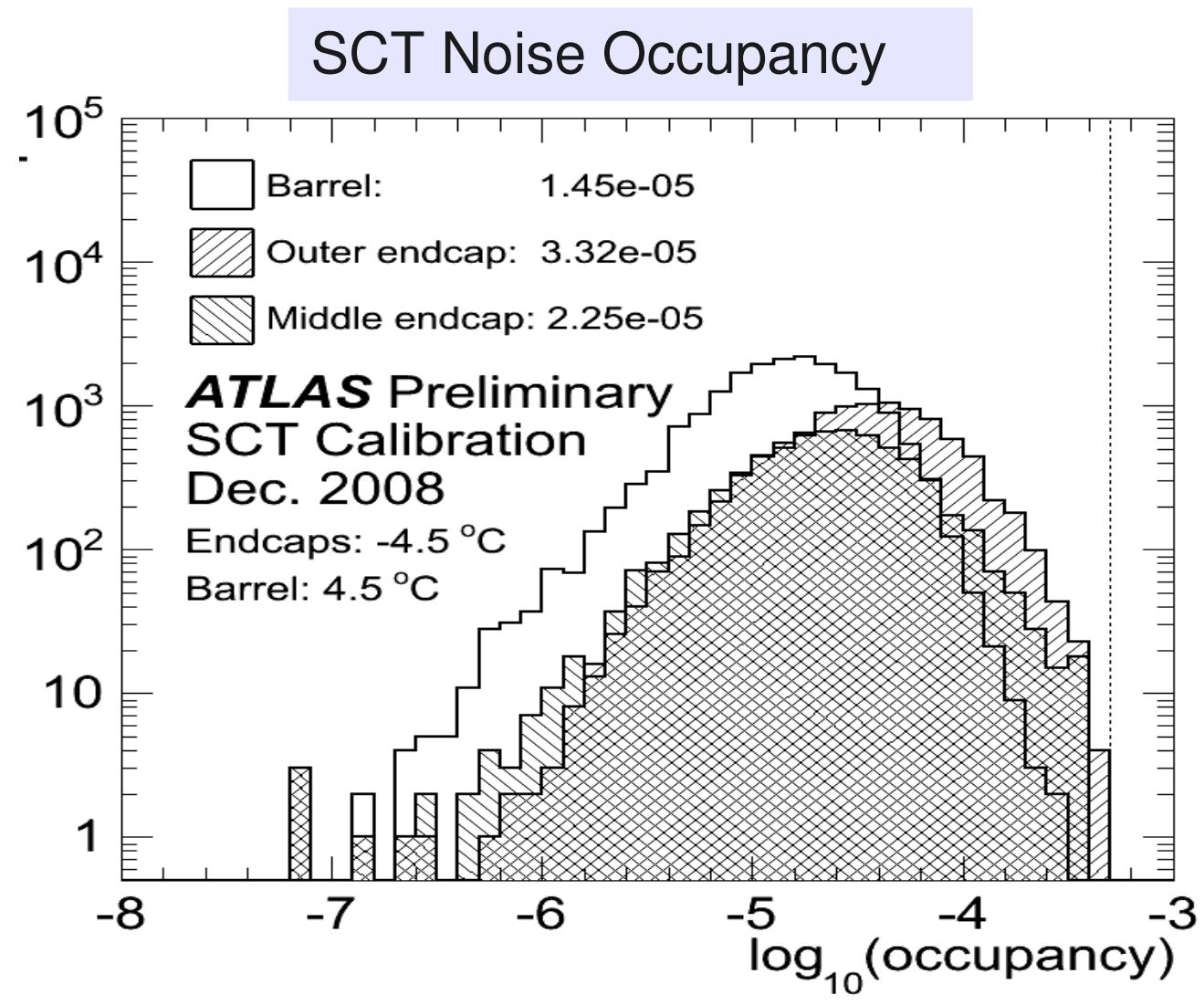

> SCT binary read-out: threshold calibration crucial !

$>$ Nominal threshold: $1 \mathrm{fC}$

$>$ Noise $2-5 \times 10^{-5}$, well below specifications: noise occupancy $<5 \times 10^{-4}$ efficiency $>99 \%$ 


\section{First Beam in ATLAS}

$>$ Pixel Detector off (safety mode)

$>$ SCT Barrel off

$>$ SCT End-caps on, for safety:

- low voltage (20 V)

- raised threshold (1.2 fC)

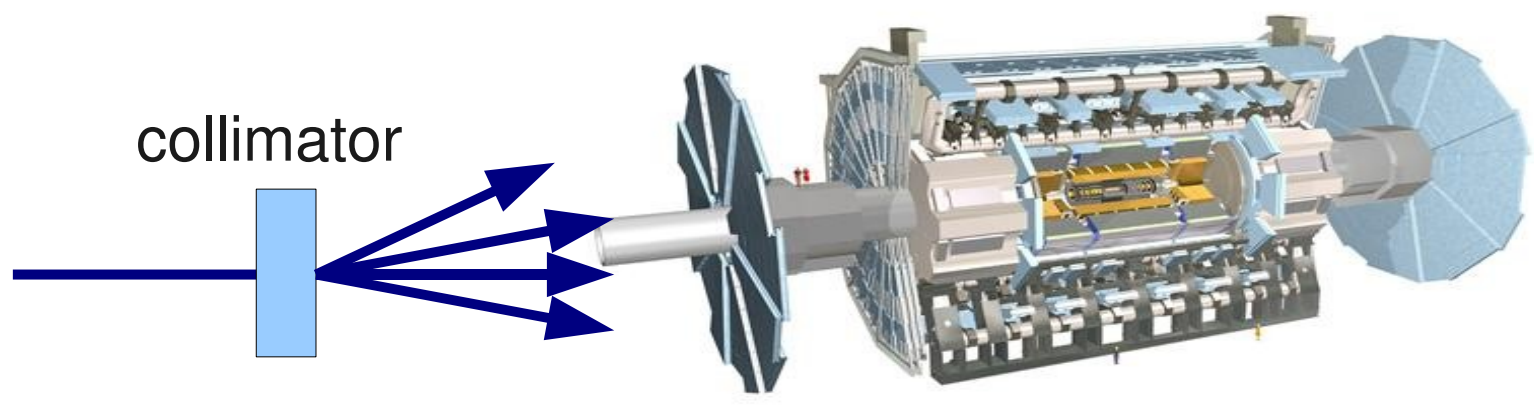

SCT EndCaps beam splash event $\quad$ 04-02-09

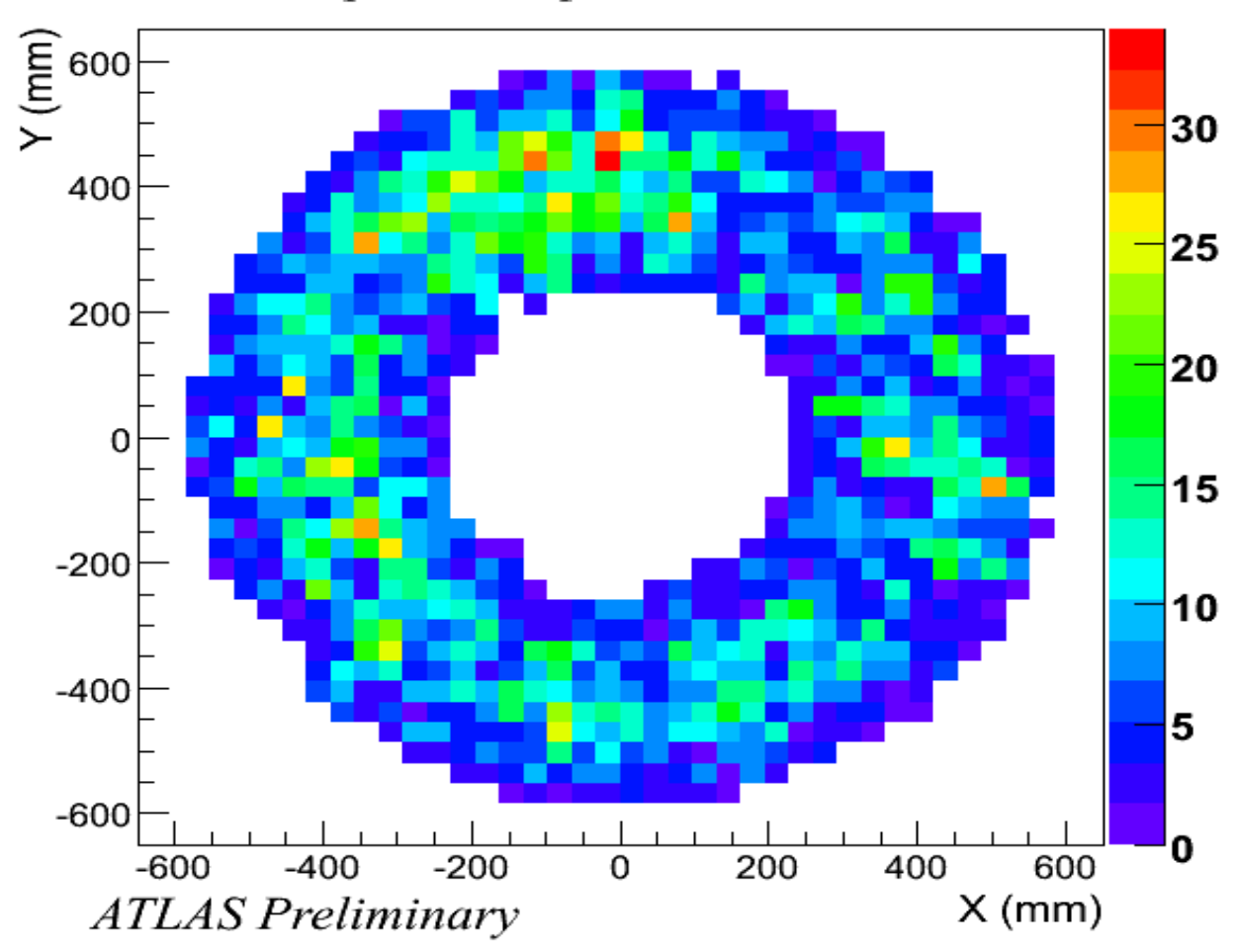

SCT EndCaps beam splash event

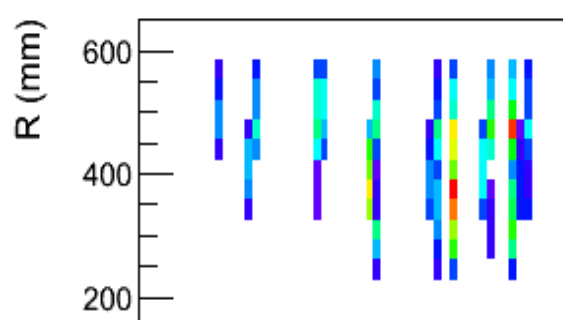

ATLAS Preliminary

04-02-09

AThas Prelinimary ard

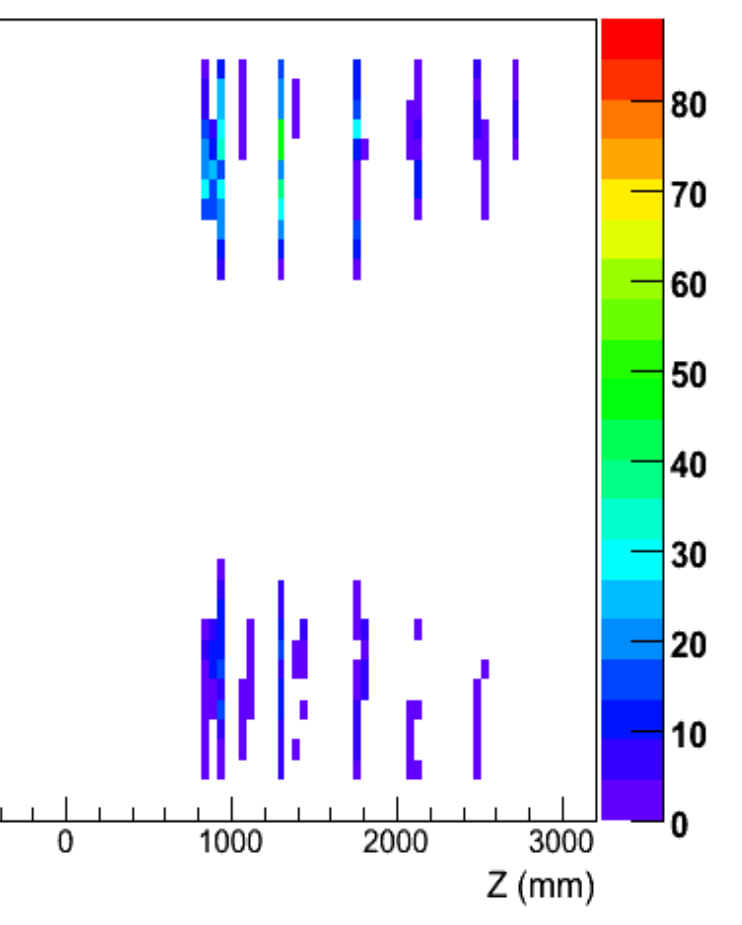




\section{Cosmics Data Taking}

$>$ Number of tracks collected with magnetic field on/off:

\begin{tabular}{|l|l|l|l|}
\cline { 2 - 4 } \multicolumn{1}{c|}{} & Field off & Field On & Total \\
\hline All track & $4.94 \mathrm{M}$ & $2.67 \mathrm{M}$ & $7.61 \mathrm{M}$ \\
\hline SCT hits & $1.15 \mathrm{M}$ & $0.88 \mathrm{M}$ & $2.03 \mathrm{M}$ \\
\hline Pixel hits & $0.23 \mathrm{M}$ & $0.19 \mathrm{M}$ & $0.42 \mathrm{M}$ \\
\hline
\end{tabular}

$>$ Fraction of operational modules:

Pixels*:

$96 \%$

${ }^{*}$ About $2 \%$ due to unavailable cooling loops, recoverable

SCT Barrel: $\quad 99 \%$ SCT EndCaps ${ }^{* *}:$ 97\%

${ }^{* *}$ Almost $2 \%$ due to unavailable cooling loops Expect $>99 \%$ operational in 2009

$>$ Cosmic runs used for:

- Timing in

- Performance studies

- Alignment

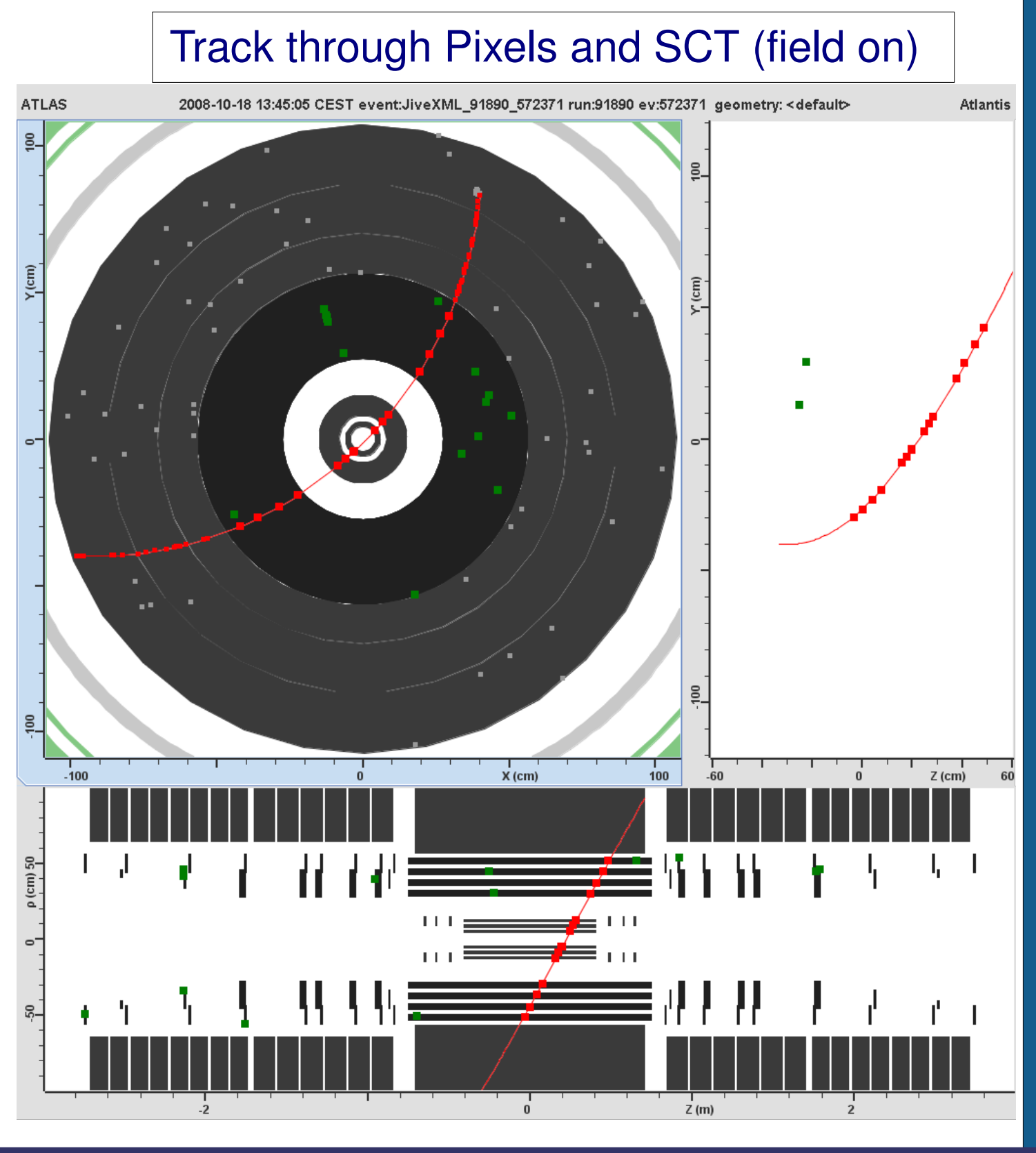




\section{Intrinsic Hit Efficiency}

Intrinsics hit efficiency from cosmic run:

$\rightarrow$ only active modules considered

$\rightarrow$ aligned geometry

Pixel Barrel

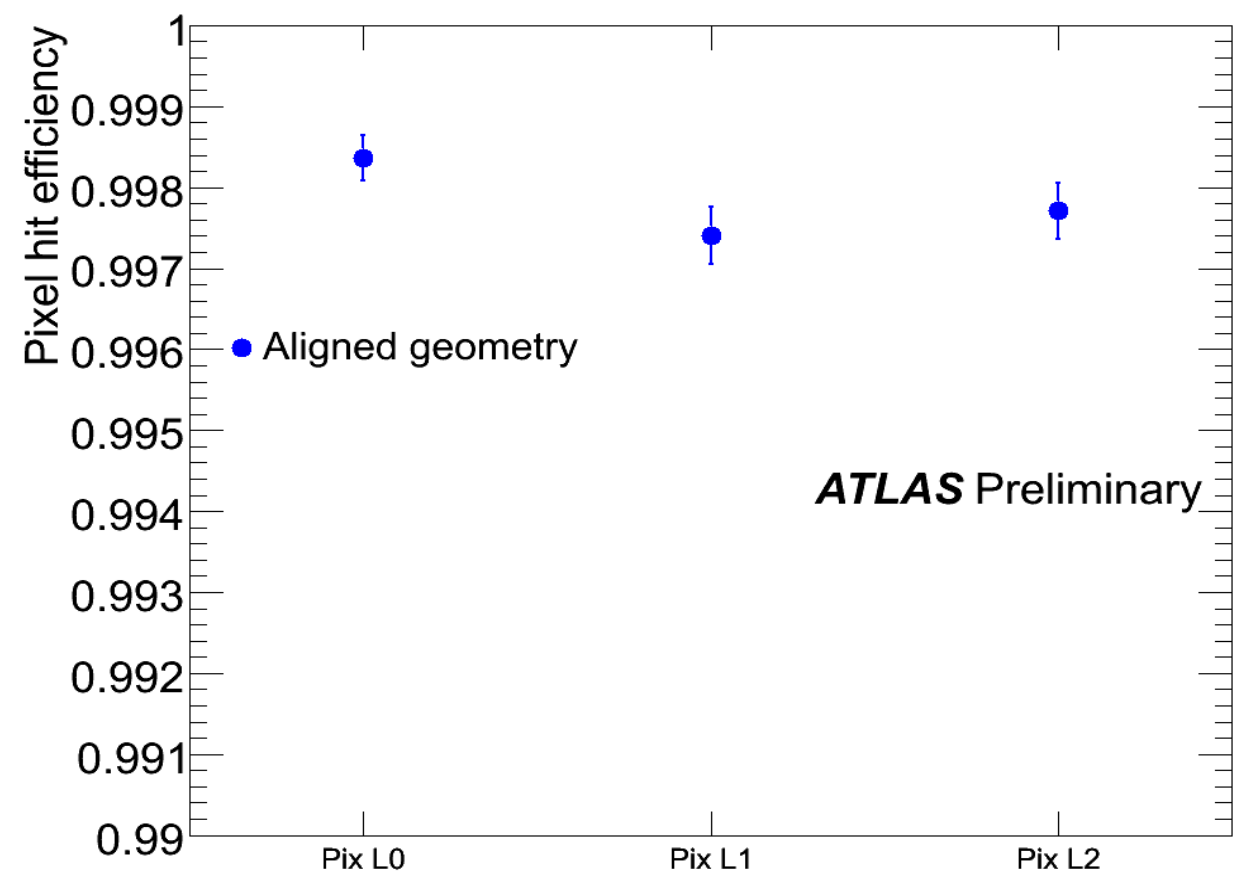

$>$ Hit efficiency per layer 99.8\%

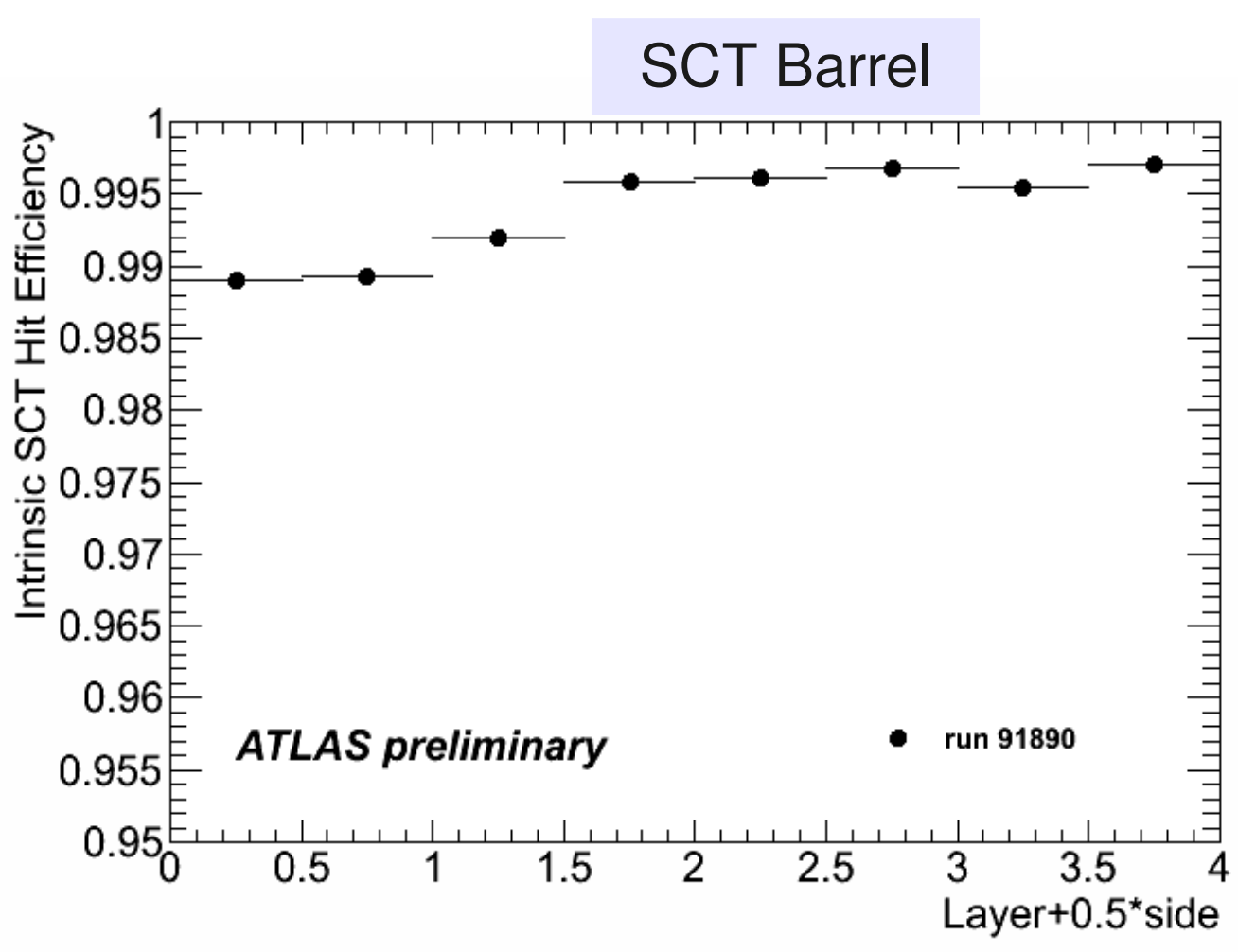

$>$ Barrel hit efficiency per layer $\geq 99 \%$ $>4$ layers with 2 sides

$>$ Incidence angle $>40$ degrees

$>$ End-caps also studied: efficiency 99\% 


\section{Timing-In the ID}

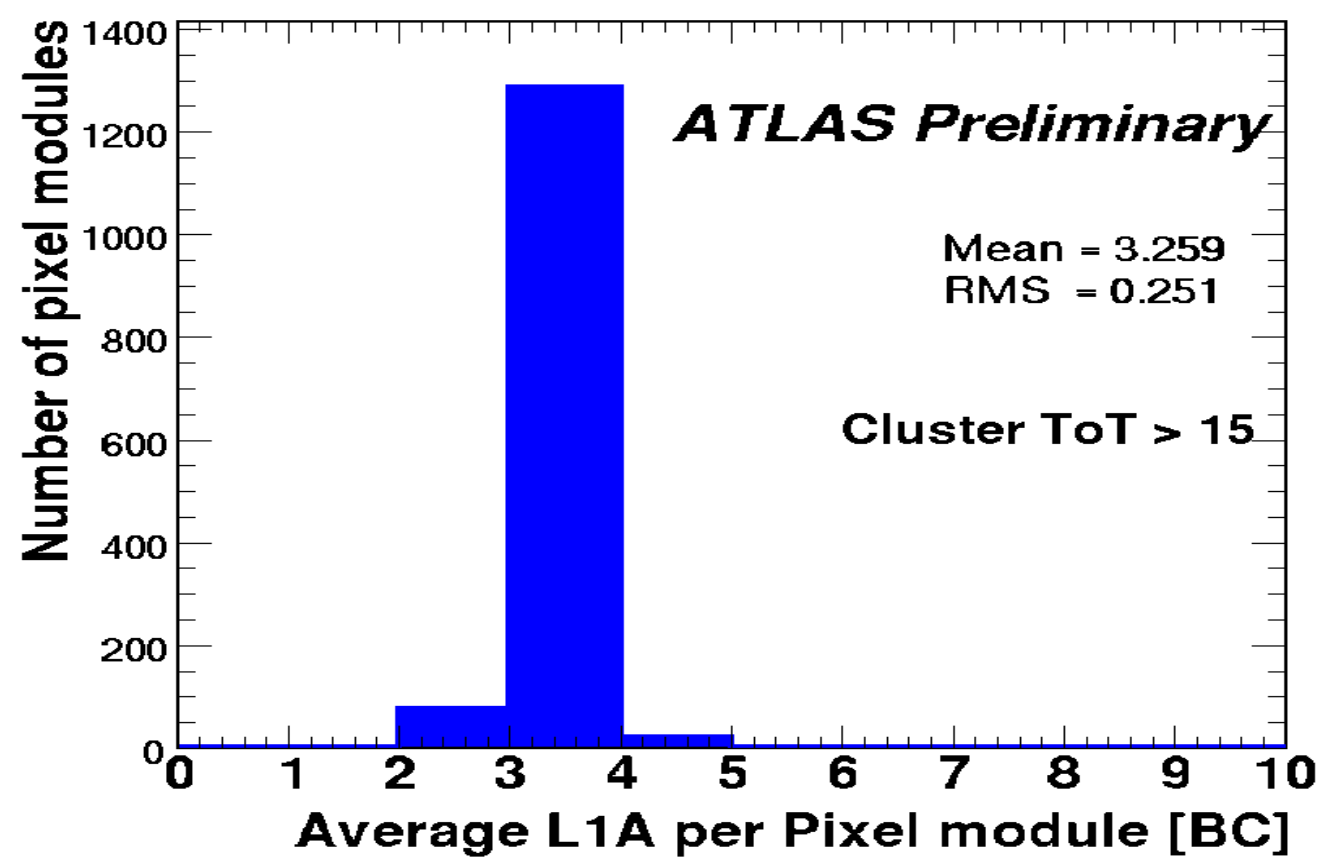

Pixels

$>$ During cosmic data-taking: read-out 8 bunch-crossings (BC)

> Plans: 5 BC (initial collisions) $1 \mathrm{BC}$ (LHC designed luminosity)

> Cluster arrival time: all modules timed-in within $\pm 1 \mathrm{BC}$

\section{SCT}

> Reads out $3 \mathrm{BC}$

$>$ SCT only track segments in barrel

> Well timed-in (within $1 \mathrm{BC}$ )

$>$ For LHC collisions: further improvements needed

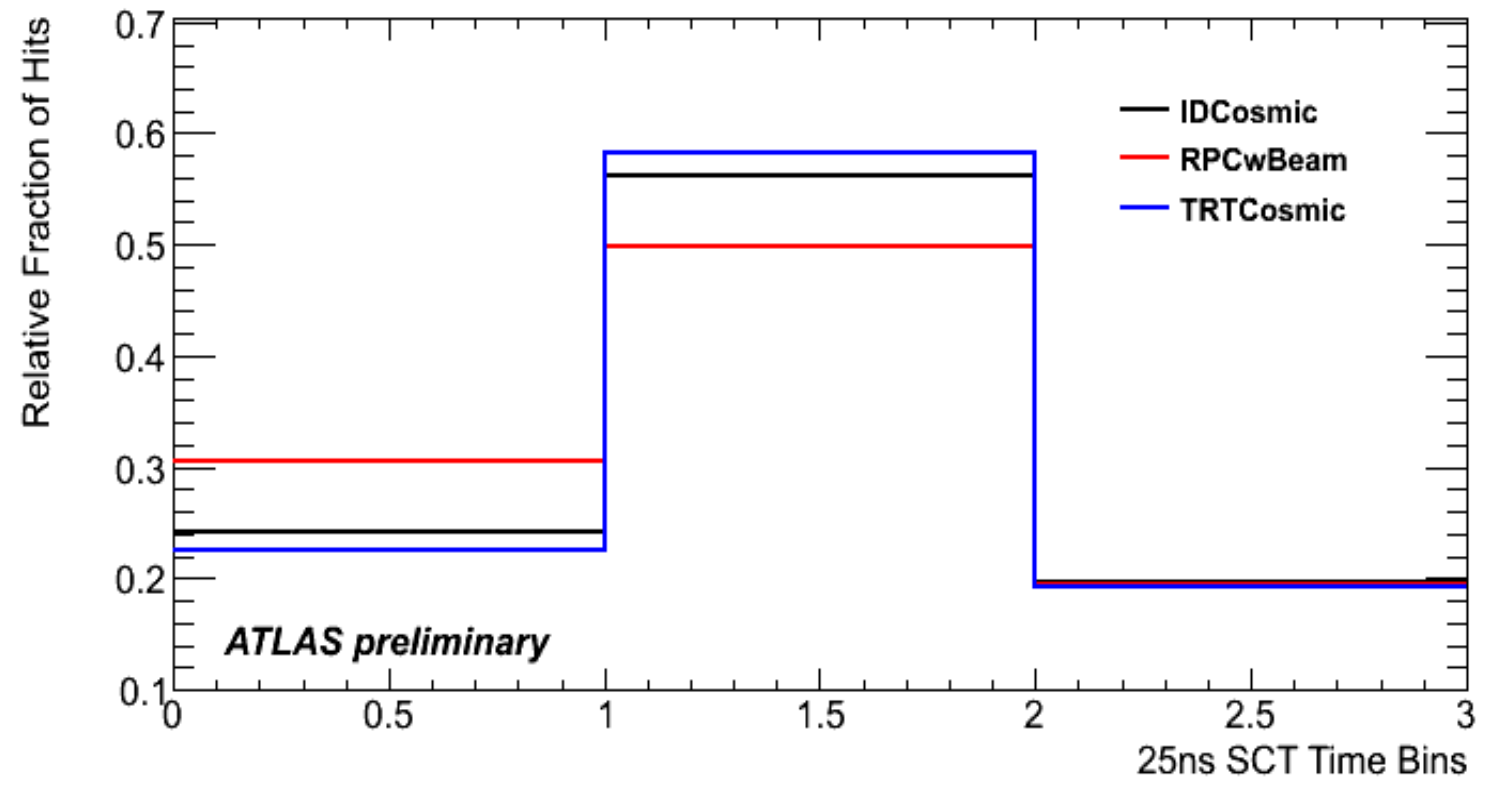




\section{Lorentz Angle}

$>$ Important to understand the detector: alignment, spatial resolution

> Dependence on $\mathbf{V}_{\text {bias }}$, temp. , external $\mathbf{B}$ field :

$>$ Measured studying cluster size vs incidence angle
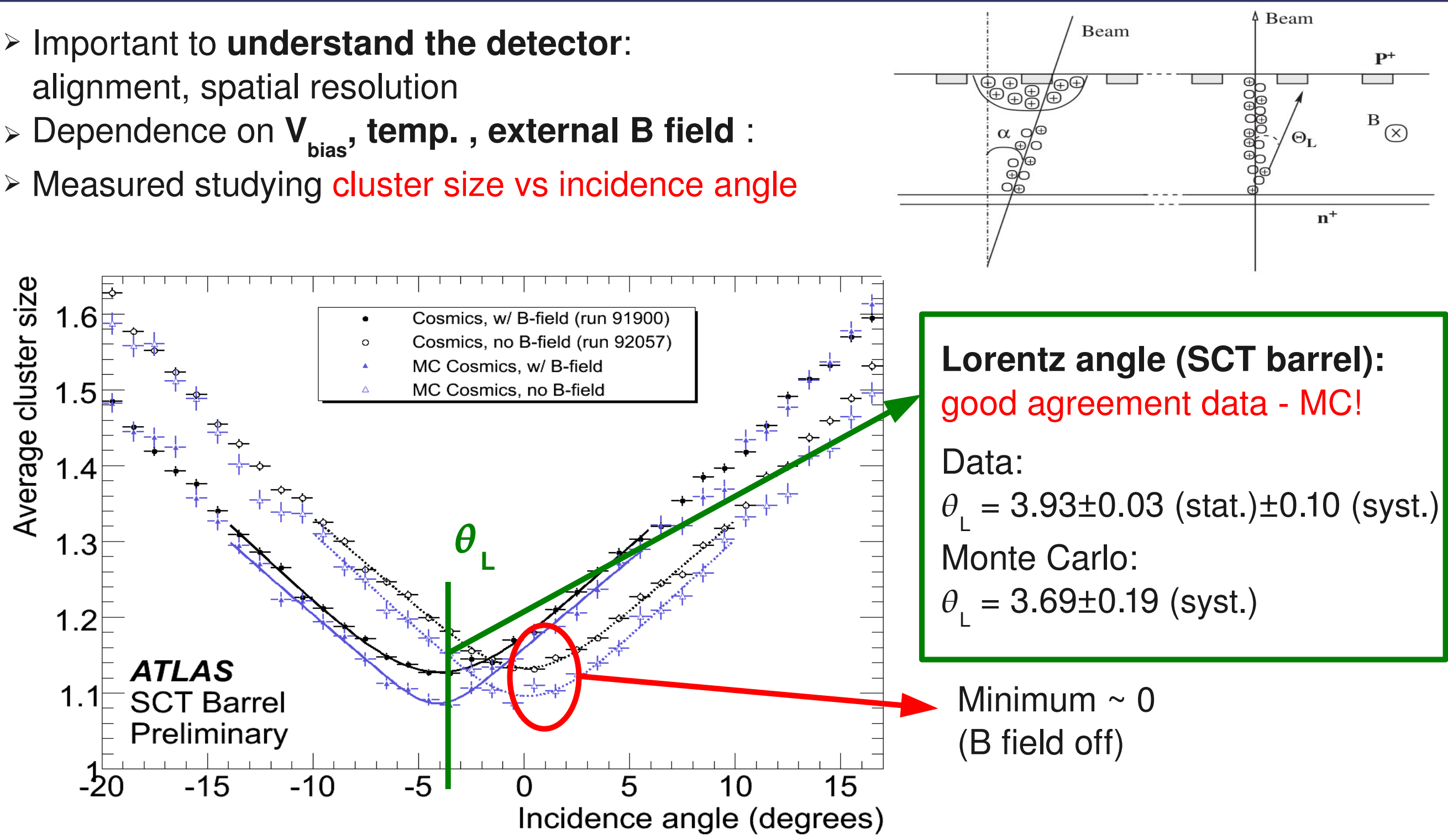

$\rightarrow$ Lorentz Angle measured also with Pixel Detector: similar results 


\section{Inner Detector Alignment}

> Barrel region aligned down to module level

$>$ End-caps: large structures aligned (too low statistics for module-to-module alignment)

Residual distribution for $x$ - coordinate in Pixels and SCT

$\rightarrow$ Great improvement in residuals: Pixels $128 \mu \mathrm{m} \rightarrow 24 \mu \mathrm{m}$, SCT $123 \mu \mathrm{m} \rightarrow 30 \mu \mathrm{m}$

$\rightarrow$ Resolution approaches the MC simulation of a fully aligned detector
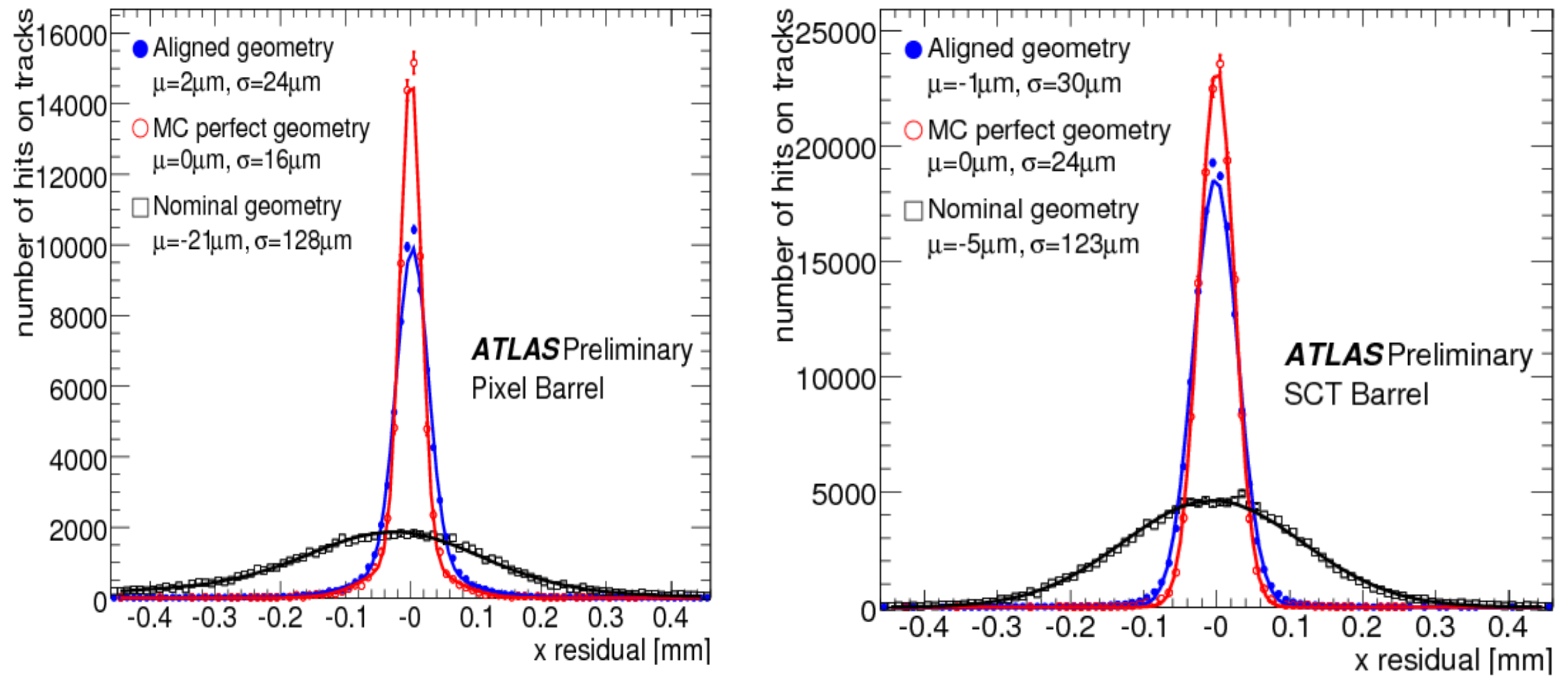


\section{Conclusions}

- Great progress in the commissioning of ATLAS Pixel and SCT Detectors in 2008

- Work to improve the detector operational reliability (evaporative cooling system and optical links):

- upgrade on cooling system in 2008/2009 shut-down period

- TX replacement on-going (until August)

- Autumn 2008: long period of calibrations and cosmic runs

- $96 \%$ of Pixels and $99 \%$ (97\%) of the SCT Barrel (End-caps) were operational

- Performance (hit efficiency and noise occupancy) within specifications

- Cosmic Data:

- High statistics (2M tracks in SCT, 400k in Pixels)

- Time-in with ATLAS, Lorentz angle measurement

- Great improvement in alignment in barrel region

- Expect $>98 \%$ of Pixels and $>\mathbf{9 9 \%}$ of SCT to be operational in 2009

- Recently (end of May) resumed operations: calibrations and cosmic runs over Summer

Excellent shape, wailting for beam in autumn 2009! 